\title{
Europas Flüchtlinge gestern und heute
}

\author{
Peter Hanenberg \\ Universidade Católica Portuguesa - Centro de Estudos de Comunicação e Cultura (CECC)
}

Zusammenfassung: Kaum ein anderer Konflikt hat das Selbstverständnis Europas als Friedensprojekt mehr erschüttert als die Kriege auf dem Balkan am Ende des 20. Jahrhundert. Noch heute sind die Wunden dieses Konflikts nicht geheilt. Deutschsprachige Schriftsteller sind vielfach in die Region gereist - jeweils aus sehr persönlichen Gründen und mit den unterschiedlichsten Absichten und Ambitionen. Zwei Texte ragen unter den Reisezeugnissen heraus: Peter Handkes "Eine winterliche Reise zu den Flüssen Donau, Save, Morawa und Drina" von 1996 und "Mein weißer Frieden" von Marica Bodrožić aus dem Jahre 2014. Eine vergleichende Lektüre der beiden Texte zeigt die historischen und politischen Gegensätze, zugleich aber auch überraschende poetische Gemeinsamkeiten.

Schlüsselbegriffe: Konflikt, Selbstverständnis Europas, Krieg, Balkan, 20. Jahrhundert, Deutschsprachige Schriftsteller, Reisezeugnis, Peter Handke, Marica Bodrožić, historisch, politisch, Gegensätze, poetisch

Resumo: Dificilmente houve outro conflito que abalasse mais o modo de pensar da Europa como projeto de paz do que as guerras nos Balcãs no final do século XX. Ainda hoje as feridas deste conflito não estão curadas. Escritores de língua alemã viajaram com frequência para esta região - cada um por razões muito pessoais e com intenções e ambições diferentes. Dois textos destacam-se entre os testemunhos de viagem: "Eine winterliche Reise zu den Flüssen Donau, Save, Morawa und Drina" de Peter Handke de 1996 e "Mein weißer Frieden" de Marica Bodrožić do ano de 2014. Uma leitura comparativa de ambos os textos mostra os contrastes históricos e políticos, mas ao mesmo tempo também mostram surpreendentemente pontos comuns poéticos.

Palavras-chave: conflito, modo de pensar da Europa, guerra, Balcãs, século XX, escritores de língua alemã, testemunhos de viagem, Peter Handke, Marica Bodrožić, histórico, político, contrastes, poético 
I.

Kaum eine andere Frage beherrscht die deutsche Öffentlichkeit zurzeit mehr als die Frage nach den Flüchtlingen. Das Thema "Reisen und andere Labyrinthe" hat damit eine Aktualität gewonnen, die so sicher nicht beabsichtigt war. Der Eintritt in immer neue bewaffnete Konflikte, die immer unerträglicher werdende Armut, die Klimabedrohungen und die mit all dem verbundenen Flucht- und Vertreibungserfahrungen machen die Erinnerung an vorangegangene Konflikte und Flüchtlingsströme besonders dringend. Auf eigentümliche Weise zeigt Heinrich Bölls Bemerkung von 1952, dass große Literatur immer schon "Kriegs-, Trümmer- und Heimkehrerliteratur" (Böll 1961: 343) gewesen ist, ihre zeitübergreifenden Relevanz. Die Erfahrung von Flucht und Vertreibung jenseits des konkreten Moments muss als eine Grunderfahrung Europas wahrgenommen werden. Der Krieg mag nicht der Vater aller Dinge sein, aber, so hat es Heiner Müller einmal formuliert, “für Europa ist der Krieg konstitutiv” (Müller 1990: 42).

Dass die aktuelle Flüchtlingsdebatte einhergeht mit einer Krise der europäischen Institutionen und des Projekts Europa im allgemeinen mag die Dringlichkeit des Themas noch erhöhen. Den Generationen der “Kriegs-, Trümmer- und Heimkehrerzeit” galt Europa ohne Grenzen als ein vielversprechendes Friedensprojekt. Nun scheint das Projekt selbst und mit ihm der Frieden in Europa auf vielfache Weise in Gefahr. Die "Renationalisierung" politischer Diskurse deutet allenthalben in eine besorgniserregende Richtung. Wenn man beispielsweise auf die deutsche Europadebatte der Gegenwart schaut, dann lassen sich zwei Positionen ausmachen, die auf den ersten Blick nicht unterschiedlicher sein könnten. Die eine Position wird vielleicht am besten von Ulrich Becks polemischem Buch von 2012 unter dem Titel Das deutsche Europa - Neue Machtlandschaften im Zeichen der Krise vertreten (Beck 2012), die andere von Herfried Münkler in seinem Buch Macht in der Mitte. Die neuen Aufgaben Deutschlands in Europa, das vor wenigen Monaten erschienen ist (Münkler 2015). Während Beck die neue zentrale Rolle Deutschlands als Machiavellismus brandmarkt und gegen ein anderes, auf Partnerschaft und Gleichberechtigung orientiertes Leitbild Europas stellt, sieht Münkler gerade in einer Stärkung Deutschlands eine Lösung für die 
Führungslosigkeit, die Europa zu überwinden habe. So unterschiedlich die beiden Positionen auch sind, sie haben gleichwohl einen gemeinsamen Nenner, nämlich die gesteigerte Aufmerksamkeit, die Deutschland im Besonderen und den Nationen im Allgemeinen in der aktuellen Europadebatte zugestanden wird.

Das zeigt sich nun in vielfacher Hinsicht als eine Last. Mit atemberaubender Geschwindigkeit werden die kostbaren Errungenschaften einer Geschichte der Überwindung von Grenzen und Differenzen zugunsten nationaler Interessen und Schutzbedürfnisse preisgegeben, als wären sie nicht die konkreten Garanten einer langen Geschichte von Frieden und Gerechtigkeit gewesen: die Lasten werden ungleich verteilt, Freizügigkeit eingeschränkt, Grenzen wiederbelebt, Abschottung zelebriert, gemeinsames Recht in Frage gestellt - und gar ein Brexit oder Grexit oder sonstwelche Austritte in Erwägung gezogen.

Wenn wir dieser Preisgabe Europas Einhalt gebieten wollen, dann sollten wir vielleicht immer wieder einmal davon Abstand nehmen, allein den Soziologen, Politikern, Journalisten und Meinungsmachern zu folgen. Mein Vorschlag war (Hanenberg 2004) und ist: Wir sollten den Schriftstellern und Schriftstellerinnen zuhören, wenn sie erzählen. Schriftsteller haben immer schon die Geschicke Europas kritisch begleitet, und zwar sowohl essayistisch als auch narrativ, d.h. mit großen erzählerischen Entwürfen europäischer Erfahrungen.

Paul Michael Lützeler hat die essayistische Auseinandersetzung (deutschsprachiger) Schriftsteller mit Europa ausführlich dokumentiert und analysiert und dabei drei besonders fruchtbare Perioden herausgearbeitet (Lützeler 1992). Die erste Periode mag als eine frühe Reaktion auf den aufkeimenden Nationalismus des 19. Jahrhunderts verstanden werden, wenn angesichts der Napoleonischen Kriege und ihrer Folgen Autoren wie Novalis, Friedrich und August Wilhelm Schlegel oder Joseph Görres ihre Visionen einer europäischen Einheit entwerfen (Lützeler 1982). Besonders lebendig wird die Debatte dann wieder im Umfeld des Ersten Weltkriegs, in der so unterschiedliche Autoren wie Annette Kolb, Hugo von Hofmannsthal, Hermann Hesse oder Heinrich und Thomas Mann dringliche Plädoyers einer europäischen Verständigung vorlegen - wobei Henrich Mann mit 
seinem Vorschlag für die "Vereinigten Staaten von Europa" ein für viele heute noch wegweisend erscheinendes Schlagwort geprägt hat (Lützeler 1987). "Achtung Europa" schrieb sein Bruder Thomas dann in den 30er Jahren als der Nationalsozialismus eine nie gekannte Bedrohung des Friedens zu provozieren begann, die schließlich nach 1945 in eine neue Welle von Europa-Plädoyers mündete, deren Erben wir heute noch sind. Es sind also die Kriege und die mit den Kriegen verbundenen Vertreibungen und Zerstörungen, die den Diskurs über Europa als Friedensprojekt immer wieder neu motiviert haben.

Und das gilt nicht nur in essayistischer Hinsicht. Der erste große Kriegsroman der deutschen Literatur ist vielleicht Grimmelshausens Abentheuerlicher Simplicissimus, dessen Geschichte die Erfahrung von Krieg, Vertreibung und Flucht literarisch erfahrbar gemacht und kritisch zur Schau gestellt hat (Hanenberg 2004: 23-48). Und seit Simplicissimus' Klage über das kriegerische, gewaltsame Europa gehört die Darstellung von Gewalt, Vertreibung und Krieg zu den unentwegten Motoren literarischer Gestaltung und Kritik. Vielleicht ließe sich - im Sinne Heinrich Bölls - durchaus behaupten, dass die europäische Literatur schon immer "Nachkriegsliteratur" gewesen ist, schon allein deshalb, weil jede Generation die Erfahrung "ihres" Krieges literarisch zur Aufarbeitung bringen musste. Dass man in der Literaturgeschichte der europäischen Perspektive in dieser Aufarbeitung $\mathrm{zu}$ wenig Aufmerksamkeit geschenkt hat, habe ich vielfältig beklagt: sei es mit Blick auf Schnabels utopischen Roman Insel Felsenburg (Hanenberg 2000), sei es mit Blick auf Lessings Nathan der Weise (Hanenberg 2008: 21) oder Goethes Faust (Hanenberg 2009), in bezug auf Kleists Antikenrezeption oder Ernst Willkomms' Die Europamüden (Hanenberg 2004: 4980). Und auch in der so genannten Gegenwartsliteratur werden die europäischen Erfahrungen, die den literarischen Entwürfen zugrunde liegen, oft nicht deutlich genug betont: sei es bei Hans Magnus Enzensberger, Günter Grass, Uwe Johnson, Robert Menasse, Cees Nooteboom, Michael Köhlmeier, Karl-Markus Gauß oder Hans Joachim Schädlich, um nur solche Autoren zu nennen, die in unserem Band Der literarische Europa-Diskurs verhandelt werden (Hanenberg/Gil 2013).

Gerne möchte ich diese Liste hier nun um drei weitere Beispiele ergänzen, die mir besonders aussagekräftig zu sein scheinen für die Frage nach Europas Flüchtlingen gestern 
und heute, eine Frage die Europas innere Verfassung ebenso betrifft wie seine weltgeschichtliche Rolle.

\section{II.}

Eine Autorin, die besonders viel zum Thema zu sagen hat, ist ohne Zweifel Marica Bodrožić, deren Buch Mein weißer Frieden 2014 erschienen ist. Marica Bodrožić ist im Alter von 9 Jahren von Dalmatien nach Hessen gezogen, noch bevor nur "acht Jahre später" (Bodrožić 2014: 1267) ein Krieg ausbrach, der zwischen 1991 und 2001 die Länder des ehemaligen Jugoslawiens überzog.

Über zehn Jahre danach macht sich Marica Bodrožić auf den Weg in ihre alte Heimat, um Orte und Menschen wiederzusehen, vor allem aber, um ihre Geschichten zu hören. Dabei entsteht ein Bild einer 'Nachkriegszeit', die man in Westeuropa gerne schon zur Vergangenheit rechnen mochte und die sich hier gleichwohl als eine immer noch zentrale Erfahrung der Gegenwart erweist. "Die jugoslawische Tragödie”, so erläutert Marica Bodrožić, "hat gezeigt, in welchem Maße die Vergangenheit in uns eingefroren ist und wie die Weltkriege in uns allen weiterarbeiten, aller äußeren Sattheit zum Trotz, führen sie zu neuen Kriegen” (Bodrožić 2014: 1399). Der “Denkraum der Nachkriegszeit” (Bodrožić 2014: 392) verlangt nach Zuhören, Dialog und einem Begriff von Heimat, der sich nicht mit Geographie verwechselt. "Meine Heimat", bekennt die Autorin einmal, "ist die Schönheit, das Sein, ein einzelner Mensch, und keine Nation, keine Religion, kein Papier wird mich je dazu bringen, Heimatgefühle (die ich als Begrenzung empfinde) aufzubringen." (Bodrožić 2014: 735). Und gerade deshalb macht sie sich auf den Weg zu den Menschen, zu den Stätten der Kindheit und des Krieges und zu den Friedhöfen und Gärten. Dort sind jene Geschichten zu erfahren, die der Gegenwart wie Spuren eingeschrieben sind. "Die Negative der europäischen Schatten", so schreibt sie, "liegen noch immer in den Dunkelkammern unserer Geschichte. Jeder ist auf seine Weise beteiligt und den dunklen Flecken etwa in der eigenen familiären Umgebung ausgeliefert. Wir stellen keine Fragen, weil wir Angst vor unvollkommenen Menschen und vor unserer eigenen Fehlbarkeit haben. Wer aber Fragen stellen kann, ist sich nah, er ist verletzbar und zeigt, dass er bereit für die Eroberung der 
Lücken im eigenen Inneren ist, bereit auch, die Welt auszuhalten, bevor sie einen neuen Namen hat." (Bodrožić 2014: 1437) Fragen stellen und die Welt aushalten, "bevor sie einen neuen Namen hat", das ist jene archäologische Arbeit, die aufzunehmen ist, wenn die Abfolge von Hass und Gegenhass, von Macht und Krieg überhaupt einmal zu unterbrechen sein soll. "Solange nicht alle Erfahrungsebenen durchdrungen sind", erkennt Marica Bodrožić, "ist keine wirkliche Freiheit möglich. Im ehemaligen Jugoslawien wie in Europa geht es stattdessen wieder nur um die sichtbare und materielle Welt, in der nur das Geld wertvoll ist" (Bodrožić 2014: 1618).

Der Autorin geht es um den - wie sie es nennt - "spirituellen Aspekt der Solidarität" (Bodrožić 2014: 1624), um ein "Dazwischen”, das sich "im Innen befindet, dass ich selbst dadurch ungebunden und frei bin, verwurzelt in den Synergien der Gleichzeitigkeit, verwandt mit der Luft und den Verwandlungen des Lebens. Sie sind mein weißer Frieden, mein Kern, den niemand sich aneignen, den niemand erobern, den niemand töten kann. Im Kern des Kerns ist mein Leben. Ich brauche keine Heimat, weil ich ein Selbst habe" (Bodrožić 2014: 1962).

Dieser weiße Frieden, das ist Marica Bodrožićs Europa, ein Europa der Begegnung, des Dialogs, des Lebens. Es ist vielleicht ein besonders schönes Zeugnis der poetischen Wahrhaftigkeit, dass sich Marica Bodrožićs Buch an dieser Stelle mit dem Zeugnis eines anderen Wanderers durch ehemals jugoslawische Landstriche trifft, der vom tagespolitischen Standpunkt aus eine entgegengesetzte Geographie betreten hat: Peter Handkes winterliche Reise zu den Flüssen Donau, Save, Morawa und Drina oder Gerechtigkeit für Serbien von 1996. Denn beide Bücher treffen sich in jenem "Dazwischen", das die Gegensätze sichtbar macht und sie doch miteinander verbindet.

"Kommst du jetzt mit dem Poetischen?" fragt Handke am Ende seines Buches, um dann zu bekennen: "Ja, wenn dieses als das gerade Gegenteil verstanden wird vom Nebulösen. Oder sag statt 'das Poetische' besser das Verbindende, das Umfassende - den Anstoß zum gemeinsamen Erinnern, als der einzigen Versöhnungsmöglichkeit, für die zweite, die gemeinsame Kindheit" (Handke 1996: 133).

Ein solcher weißer Frieden, eine zweite Kindheit ist kein politisches Programm, auch 
keine großartige Idee und kein demokratiekritischer Protest. Es ist vielmehr der lebendige Ausdruck einer gelebten Erfahrung - "Erdung und Rettung" (Bodrožić 2014: 3606) in einem. "Ich habe keine Lehre, aber ich führe ein Gespräch." zitiert Bodrožić Martin Buber als wäre das der Schlüssel zum mündigen Menschen und zum gelingenden Europa.

Marica Bodrožićs Europa ist eine Gestalt eigenen Rechts, gegründet in der Erzählung und im Dialog, gegründet aber auch in der Erfahrung der verlassenen Heimat und in der Erfahrung der Reise. Beide, die verlassene Heimat und die Reise, gehören in ihrer Dynamik also auch hier zu den konstanten Merkmalen europäischer Erzählungen, sie sind in gewisser Weise der Stoff, aus dem Europa entsteht. Nicht Heimat und Nation, weder Deutschland noch Brüssel können wirklich Fixpunkte europäischer Erfahrung sein. Die Spannung zwischen Nähe und Ferne markieren vielmehr ein Dazwischen, mit dem Europa immer wieder über sich selbst hinausgewachsen ist.

III.

Die Spannung zwischen Nähe und Ferne und die Erfahrung von Flucht und Vertreibung bestimmen auch die Geschichte von Simone Grolmann, ihren Eltern und Großeltern, ihrer Tochter und ihren Freunden, wie sie von Ulrike Draesner in ihrem großartigen Roman Sieben Sprünge vom Rand der Welt aufgeschrieben worden ist. Simone Grolmann ist Verhaltensforscherin, schon ihr Vater hatte eine erfolgreiche Karriere als Wissenschaftler gemacht und kann und kann von seinen Primaten nicht lassen. Den Vater interessierte "das Gehirn", die Tochter interessieren "Gehirn und Verhalten" - und damit stellen beide die heute zentralen und dringenden Fragen nach der condicio humana: nach der Freiheit des Willens etwa oder nach der Empathie, der Fähigkeit des Menschen, die eigentlich ein Zwang, eine Falle ist, "eine neurologische Zwangsjacke". Und schließlich die Kernfrage überhaupt, wie sie Frau Prof. Grolmann in ihrer Vorlesung formuliert:

Die Frage, um die es in Zukunft gehen wird, weit über die Primatenforschung hinaus, die Frage, die wir brauchen, um uns in Zukunft verantwortlich und sinnvoll auf diesem Planeten zu bewegen, um Konzepte von Zukunft überhaupt zu entwickeln, die Frage, bei deren Beantwortung uns die Affen 
helfen, diese Frage, meine Damen und Herren, lautet: Wo - wo sind wir zuhause? (Draesner 2014: 1644)

"Wo sind wir zuhause?" - diese Frage muss jeder der Protagonisten anders beantworten, sei es Prof. Simone Grolmann selbst, sei es ihr Vater Eustachius Grolmann oder ihre Tochter Esther, sei es Lilly, die Großmutter, oder Hannes, der Großvater, Soldat in zwei Weltkriegen, oder deren älterer Sohn Emil, der zwar trotz seiner Behinderung dem Euthanasie-Programm entgeht, dann aber doch auf der Flucht zu Tode kommt. Oder Boris Nienalt, der Psychologe, der über posttraumatische Störungen forscht, sich der Familie Grolmann annimmt und ein Buch unter dem Titel “Das geteilte Gedächtnis" veröffentlicht, sei es Jennifer, seine Tochter oder Halka, seine Mutter. All diesen Figuren ist ein eigenes Kapitel gewidmet, der Roman spricht mit und aus der Perspektive dieser Figuren, gibt ihnen Stimme, setzt sie in ihr Recht. So wie Lilly, die Großmutter, die auf der Flucht ihren behinderten Sohn verliert und die nicht wieder zurückfindet in eine geordnete Sprache, deren Erinnerungsstücke sich lesen wie Gedichte und doch voller Geschichte sind: "unwiedergutbar" stempeln sich diese "seelische[n] Landschaften" "von einer Generation in die nächste hinüber" (Draesner 2014: 101), wie es der fiktive Psychologe Boris formuliert.

Zentrales Thema dieser Erinnerung ist die Vertreibung - ein Thema, das den Roman durchzieht wie die Generationen, die in ihm zur Sprache kommen. Vertreibung hat den Protagonisten “den Rahmen verzogen [...], auch den Kindern noch” (Draesner 2014: 5277). "Stell dir Vertreibung", so heißt es einmal, "als ein Gefängnis vor, nur umgekehrt: Man rennt, statt eingesperrt zu sein. Beide Male wird auf dieselbe Weise gerechnet, du wirst an einen anderen Ort gezwungen, nur dass Vertreibung viel billiger ist" (Draesner 2014: 5283).

Jede Generation begegnet der Vertreibung in der je aktuellen Gestalt: "Flucht, Vertreibung, Zwangsverschleppung, Asyl" (Draesner 2014: 6788), so heißt es für die Gegenwart, derart, dass Lillys Urenkelin ein Projekt betreibt zur Erforschung geleugneter Migration: "Liebesmigration, Mischehen und internationale Patchworkfamilien interessierten uns ebenfalls, auch in diesen Fällen stritten die Beteiligten die 
Migrationsbewegung überdurchschnittlich häufig ab” (Draesner 2014: 6834). Und so heißt es einmal mit Blick auf die Gegenwart: "Seit 2000 wanderten die Menschen stärker als die Vögel” (Draesner 2014: 7176).

Heimat ist ein sehr vorläufiger Begriff - heute wie in früheren Zeiten von Flucht, Vertreibung und Migration. Die historische Gestalt der darin enthaltenen Erfahrung mag variieren, die Herausforderung als solche bleibt sich gleich. Vielleicht hat Eustachius Grolmann deshalb darauf bestanden, dass seine Enkelin den Küchentisch als Erbe annimmt: "An ihm sollten wir sitzen" (Draesner 2014: 7315). Und reden und uns von unseren Wegen und Orten erzählen.

\section{IV.}

Europa ist eine Erfahrung im Raum und in der Zeit, Orte lassen sich finden und werden verloren, Generationen hinterlassen ihre Spuren, überlassen ein stets neu $\mathrm{zu}$ erzählendes Erbe auf der Suche nach Frieden. Das gilt auch für ein Buch von Botho Strauß, das scheinbar weder auf die Erfahrung der Vertreibung noch des Krieges gegründet ist. Botho Strauß ist ein Autor ohne 'Migrationshintergrund', zu jung für den alten, zu alt für den heutigen Krieg, so könnte man meinen.

Doch Botho Strauß' überraschend persönliches Buch Herkunft aus dem Jahre 2012 antwortet auf die gleiche Frage, die auch Marica Bodrožićs und Ulrike Draesners Büchern zugrunde liegt: Wo sind wir zuhause? Und auch hier sind es Krieg und Vertreibung, die als übermächtige Erfahrung die Erzählung bestimmen, nämlich der Erste Weltkrieg, in dem der Vater verwundet wurde, und die Flucht aus dem Osten vor der politischen Verfolgung im Nachkriegsdeutschland. Der Sohn hingegen hat "in den vergangenen sechzig Jahren ausschließlich in Friedensgesichter geschaut, täglich Friedensverrichtungen ausgeführt, in Friedenszimmern gesessen und Friedensaussichten genossen". Dieser Kontrast in den Erfahrungen der Generationen ist zugleich ihr starkes Band. "Wir sind, wie wir da sind, von gestern her", zitiert Botho Strauß das Buch Hiob, "und unsere Erinnerung ist lediglich ein Ausscheidungsprodukt des alle Gegenwart vertilgenden Einst." (Strauß 2014: 722). "Das Gedächtnis", so bekennt Strauß, "ist eine Variable der Sehnsucht, so daß Fernweh und 
Heimweh, Erwartung und Erinnerung in ein und demselben "Enzym« des Unerreichlichen symmetrisch angeordnet sind" (Strauß 2014: 325). Und an anderer Stelle "Alle Bewegung der Erinnerung gleicht Perihel und Aphel des Planetenlaufs um die Sonne: Herkunftsnähe, Herkunftsferne. Nie aber wird der Umlauf verlassen" (Strauß 2014: 407). Wo aber, so könnte man fragen, hat der Umlauf seinen Ort, wenn auf ihm Nähe und Ferne gleichermaßen zu erreichen sind? Und vielleicht könnte man auf diese Frage mit einer anderen antworten, die auch Botho Strauß sich stellt: "War nicht alles im wesentlichen Gespräch?" (Strauß 2014: 448).

V.

Blicken wir von den aktuellen Erfahrungen von Flucht und Vertreibung auf diese literarischen Werke, dann sehen wir, wie kostbar und schwierig, wie alt und wie immer neu, wie allgemein und wie konkret diese Erfahrungen sind: ohne sie gäbe es Europa und nicht, nicht seine Anziehungskraft, nicht seinen Frieden. Es wird neuer Geschichten bedürfen, wenn wir verstehen wollen, was heute geschieht. Ob Bodrožićs' weißer Frieden, Handkes zweite Kindheit, Draesner sieben Sprünge oder Botho Strauß' Herkunft: sie erzählen von einem Leben in Europa, das auf Flucht und Vertreibung ebenso gründet wie auf der Sehnsucht nach Frieden. Die Erinnerung an Krieg, Flucht und Vertreibung von einst ist der notwendige Horizont gegenwärtiger Herausforderungen. Und Schriftsteller waren und sind zuverlässige Garanten eines Dialogs und einer Mühe um Verständigung, die heute so wichtig und nötig ist wie gestern (Hanenberg 2016). Es ist keine bloße Idee und mehr als ein Programm, ein Gespräch zwischen Generationen, aus der Erfahrung von Krieg und Frieden, Flucht und Vertreibung. Europa und die Flüchtlinge: sie gehören zusammen.

Vielleicht verhält es sich am Ende mit Europa wie mit Marica Bodrožićs Strickjacke aus Kindertagen: "dass nicht meine blaue Strickjacke kleiner, sondern ich selbst größer wurde. Auch das hatte mir niemand erklärt" (Bodrožić 2014: 136): bis der Satz selbst die Erfahrung in Erkenntnis verwandelt. 


\section{Bibliografie}

Beck, Ulrich (2012), Das deutsche Europa: Neue Machtlandschaften im Zeichen der Krise, Berlin, Suhrkamp.

Bodrožić, Marica (2014), Mein weißer Frieden, München, Luchterhand, Kindle-Edition.

Draesner, Ulrike (2014), Sieben Sprünge vom Rand der Welt: Roman, München, Luchterhand, Kindle-Edition.

Böll Heinrich (1961), Erzählungen, Hörspiele, Aufsätze, Köln, Kiepenheuer \& Witsch.

Handke, Peter (1996), Eine winterliche Reise zu den Flüssen Donau, Save, Morawa und Drina, oder, Gerechtigkeit für Serbien, Frankfurt am Main, Suhrkamp.

Hanenberg, Peter (2000), "Eine deutsch-portugiesische Kulturbegegnung in indischen Meeren. Zu Johann Gottfried Schnabels Insel Felsenburg”, in Helmut Siepmann (Coord.), Portugal, Indien und Deutschland. Portugal, Índia e Alemanha. Actas do V Encontro LusoAlemão, Köln, Lisboa: Zentrum portugiesischsprachige Welt: 171-177.

-- (2004), Europa. Gestalten. Studien und Essays, Frankfurt/M. u.a.O, Lang.

-- (2008), "Europa als literarische Erfahrung in der deutschsprachigen Literatur seit dem 17. Jahrhundert", in Christoph Parry, Liisa Voßschmidt (Hg.), Europäische Literatur auf Deutsch? Beiträge auf der 13. Internationalen Arbeitstagung Germanistische Forschungen zum Literarischen Text, Vaasa, 18.-19.5.2006, München, iudicium: 15-29.

-- (2009), “Fausto, Europa y 'el retrato de toda una cultura' de Spengler”, in Arno Gimber, Isabel Hernándes (coord.), Fausto en Europa. Visiones de los demonios y el humor fáustico, Madrid, Editorial Complutense: 147-158.

-- (2016), “Deutsch aufgeben: literarische Übungen mit Flüchtlingen”, in REAL. Revista de Estudos Alemães 6: 79-89.

--/ Isabel Capeloa Gil (2013), Der literarische Europa-Diskurs. Festschrift für Paul Michael Lützeler zum 70. Geburtstag, Würzburg: Königshausen \& Neumann. 
Peter Hanenberg

Lützeler, Paul Michael (1982), Europa. Analysen und Visionen der Romantiker, Frankfurt/M., Insel.

-- (1987), Plädoyers für Europa, Frankfurt/M., Fischer Taschenbuch Verlag.

-- (1992), Die Schriftsteller und Europa. Von der Romantik bis zur Gegenwart, München, Zürich, Piper.

Müller, Heiner (1990), "Stirb schneller Europa", in Heiner Müller, Zur Lage der Nation. Heiner Müller im Interview mit Frank M. Raddatz, Berlin, Rotbuch: 25-42.

Münkler, Herfried (2015), Macht in der Mitte die neuen Aufgaben Deutschlands in Europa, Hamburg, Körber-Stiftung.

Strauß, Botho (2014), Herkunft, München, Hanser, Kindle-Edition.

Peter Hanenberg, Studium der Germanistik und Philosophie an den Universitäten Tübingen und Bamberg, Dr. phil.; 1995-2006 Professor für Deutsche Literatur in Viseu, Portugal; seit 2006 Professor für Germanistik und Kulturwissenschaften an der Universidade Católica Portuguesa in Lissabon; 2006-2010 Vorsitzender des portugiesischen Germanistenverbands APEG; seit 2012 Leiter des kultur- und medienwissenschaftlichen Forschungszentrums CECC; Research Center for Communication and Culture, Lissabon. 